\title{
Effective Septal Extension Graft for Asian Rhinoplasty
}

\author{
Jae Hoon Kim, Jin Woo Song, Sung Wan Park, Won Suk Oh, Joo Heon Lee \\ April 31 Plastic Surgery Clinic, Seoul, Korea
}

Background This study aimed at overcoming the disadvantages of septal extension grafts and keeping the nasal tip as natural as possible by using different forms of the graft and additional supporting methods depending on the case.

Methods Among 458 patients who received a septal extension graft from March 2008 to September 2011, 107 patients were selected who underwent at least a 6-month follow-up. Patients were categorized according to the primary objective of the operation; an upturned tip correction or tip lengthening, tip lengthening with tip projection, or retracted columella correction. Each group of patients received a different type of septal extension graft out of 3 different types of grafts based on the purpose of the operation. The evaluation of the results was made from by comparing preoperative and postoperative photographs of the tip angle, projection, and nasolabial angle.

Results The average tip angle for the patients in the upturned tip correction group was reduced to 98.3 from 124.9 degrees. For the patients in the tip lengthening with tip projection group, the average tip angle was reduced to 96.8 from 122.4 degrees and the average tip projection was increased to 27.5 from $23.2 \mathrm{~mm}$. The average nasolabial angle for the patients in the retracted columella correction group was increased to 94.6 from 74.8 degrees.

Conclusions Sufficient nasal tip lengthening and projection could be achieved by applying a septal extension graft using the graft best suited for the group of patients categorized by surgical objective.

Keywords Nose / Rhinoplasty / Nasal cartilages
Correspondence: Jae Hoon Kim April 31 Plastic Surgery Clinic, 6th floor, Geonwoo B/D, 548 Gangnam-daero, Gangnam-gu, Seoul 135-010, Korea

Tel: +82-2-540-6777

Fax: +82-2-540-6222

E-mail: April31kjh@hanmail.net

Received: 10 May 2013 • Revised: 11 Jun 2013 • Accepted: 29 Jun 2013

pISSN: 2234-6163 • elSSN: 2234-6171 • http://dx.doi.org/10.5999/aps.2014.41.1.3 • Arch Plast Surg 2014;41:3-11

No potential conflict of interest relevant to this article was reported.

\section{INTRODUCTION}

The degree to which the height and length of the nasal tip can be increased and maintained to enhance the aesthetic quality of the nose is surely one of the major challenges and concerns for plastic surgeons. Furthermore, since the naturalness of the nasal tip must not be damaged, surgeons apply various methods using autologous materials to solve this problem. Unlike Caucasians,
East Asians undergo more operations extending the height and length of the nose than operations to reduce the size of the nose. The extension of East Asians' noses requires the sufficient release of soft tissue and an adequate supporting cartilage framework. The septal extension graft is a very suitable method to extend and maintain the thick skin of Asians' noses since it provides a direct extension of the framework along while maintaining strong support. However, there are many limitations for this operation 
due to Asians' comparatively low nasal height, broad bony vault, small amount of harvestable septum with respect to the shorter nose length and the weakness of alar cartilage for shaping the nasal tip. To preserve the advantages of the septal extension graft and the natural mobility of the nose as much as possible while effectively utilizing the limited amount of septum, we applied different types of septal extension grafts appropriate to each case, achieving optimal results.

\section{METHODS}

\section{Subjects}

Among the 458 patients who received a septal extension graft for the purpose of tip extension from March 2008 to September 2011, 107 patients were selected who were eligible for the 6-month follow-up period. Preoperative counseling was conducted to determine the patient's preferred nasal shape and photos taken from various angles were analyzed in order to plan the septal extension graft. Then, based on the main purpose of the operation, the patients were categorized into 3 groups: patients who needed upturned tip correction or tip lengthening were classified as group 1, those in need of upturned tip [1] correction with tip projection were considered group 2 , and patients with a retracted columella according to the classification of alarcolumellar discrepancies by Gunter et al. [2] were considered group 3. There were 41 patients in group 1, 58 in group 2 , and 8 in group 3. The average age of the patients was 29.6 years (range, 18-57 years), and they consisted of 92 female patients and 17 male patients. It was the primary surgery for 43 of them and secondary for the other 66 . The evaluation of the operation results was made by comparing the preoperative and postoperative photos of the nasal tip angle and projection (from the nasal tip to the alar crease base) measured from along with the postoperative satisfaction level of the patients. The postoperative tip projection, extension maintenance, and tip mobility were also compared to preoperative levels.

\section{Surgical technique}

Open rhinoplasty was performed in all of the cases. The skin flap was elevated by linking a columellar incision and an infracartilaginous incision. When the incision was performed along the caudal margin of the lower lateral cartilage, it was applied up to the most lateral part of the lateral crus. The elevation of the skin flap was conducted widely to make redraping of the stretched cartilage structures possible without tension.

In the primary surgery cases, the release of the cartilage structure was performed carefully so as not to damage the lower lateral cartilage and the upper lateral cartilage. In the second- ary cases, the separation of the skin flap and the scar tissue was performed carefully and the attachment of a portion of the scar to the cartilage was conducted in the cases of severe cartilage damage to make a more symmetrical form of the alar cartilages possible.

Free movement without tension of the lower lateral cartilage was made by complete dissection of the scar tissue or the support structure which was holding the tip structure firmly. Under the traction of the lower lateral cartilage, the pyriform ligament of the scroll area between the upper lateral cartilage and lower lateral cartilage was sufficiently dissected, taking care not to damage the nasal mucosa, allowing free movement without tension of the dormal segment of middle crus and the tip. Not only better derotation but also better advancement can be achieved when the nasal hinge is separated such that a full release of the pyriform ligament around the lateral crus of the lower lateral cartilage is given by more dissection to the lateral, allowing the lateral crus to be completely separated from the structure of the pyriform aperture (Figs. 1, 2) [3-6]. Through these processes, an effective tip projection and derotation without tension were achieved.

The septal cartilage was harvested by using a caudal septal approach, leaving a 8 - to 12 -mm L-shaped strut, depending on the thickness of the septum. The harvest around the keystone part and the caudal angle part was made gently with a smooth curve, and the keystone part and the caudal angle part were bluntly removed. To harvest the maximum septal cartilage within a safe range, it was harvested after the careful dissection of the maxillary crest of the lower portion and the vomer part, with caution not to damage the mucosa. The bony septum was carefully in-

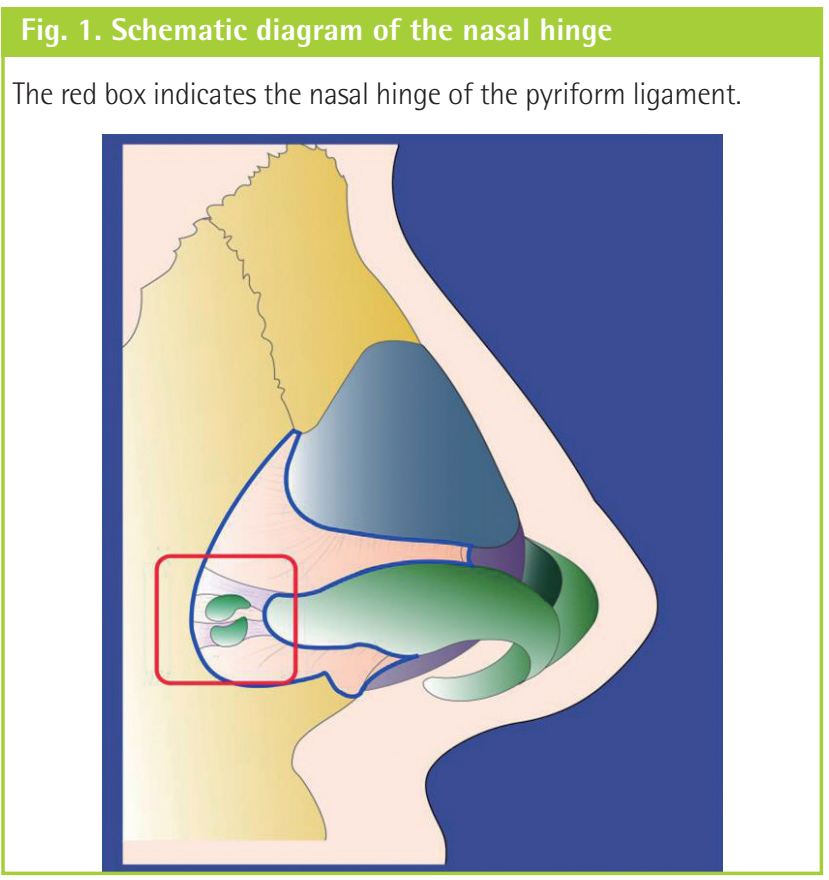




\section{Fig. 2. Complete release of the pyriform ligament}

$(A, B)$ Photographs demonstrating free movement of the nasal tip without tension by a complete release of the lower lateral cartilage.
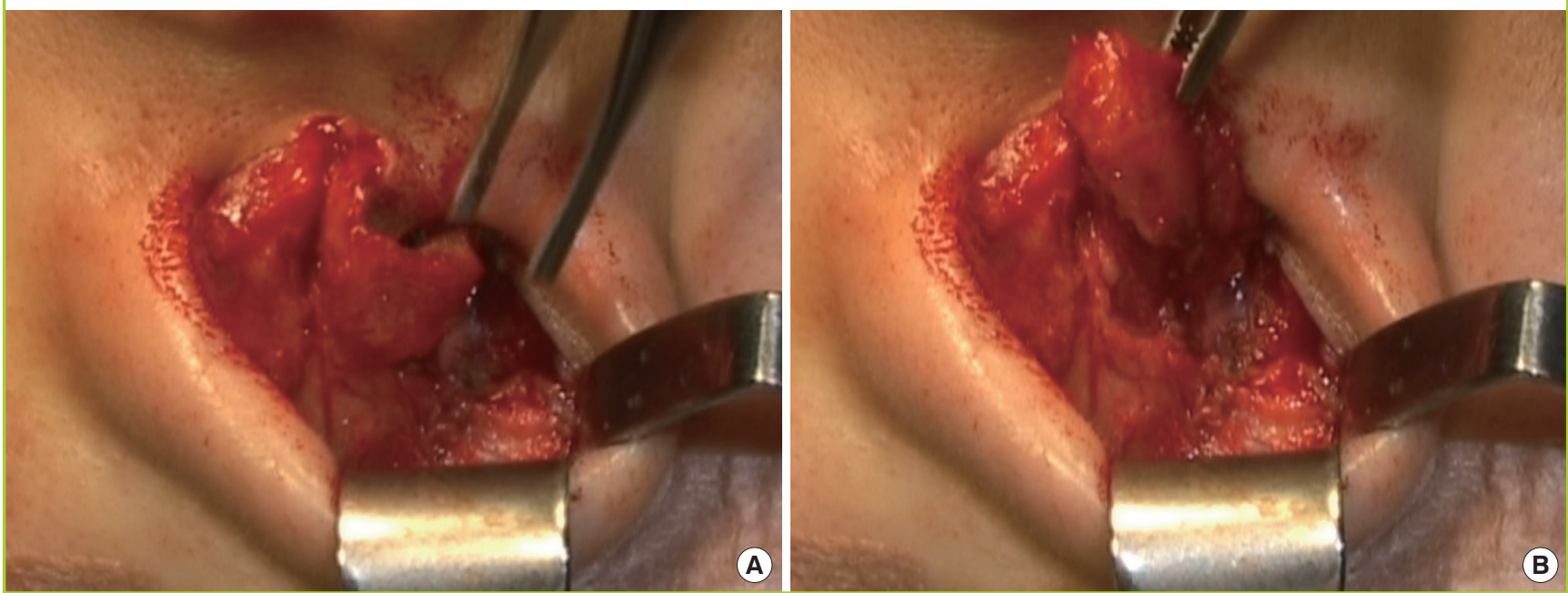

\section{Fig. 3. Type I septal extension graft}

(A) Schematic diagram and (B) photograph of type I septal extension graft. The type I septal extension graft is designed to preserve the lower portion of the membranous septum.
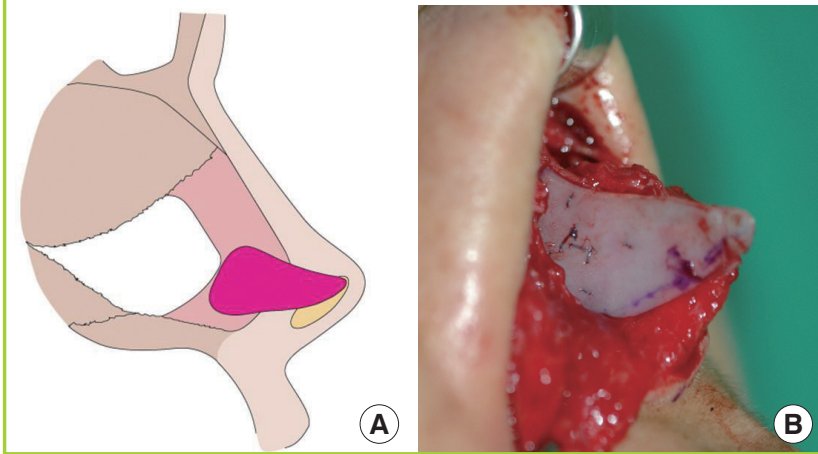

cluded in the harvest as necessary.

Three types of septal cartilage grafts were used, depending on the purpose of the operation. First, a type I septal extension graft was used for cases of upturned tip correction to conserve the lower portion of the membranous septum as much as possible (Fig. 3). This allowed for the maintenance of mobility and naturalness of the nasal tip and the left-over graft could be utilized in various ways such as the dorsal batten graft for the reinforcement of support or the tip onlay graft. Next, a type II septal extension graft was used for cases in need of tip derotation and tip projection at the same time so that the graft could sufficiently cover all the way down to the nasal base (Fig. 4). The graft was allotted to the nasal base, depending on whether the tip derotation or tip projection would be the main objective of the operation. In the cases of allotting the cartilage down to the nasal base to maintain the nose height, the amount of the graft

\section{Fig. 4. Type II septal extension graft}

(A) Schematic diagram and (B) photograph of type II septal extension graft. To maintain nasal tip projection, the septal extension graft should be placed above the nasal floor, but should not reach the anterior nasal spine.
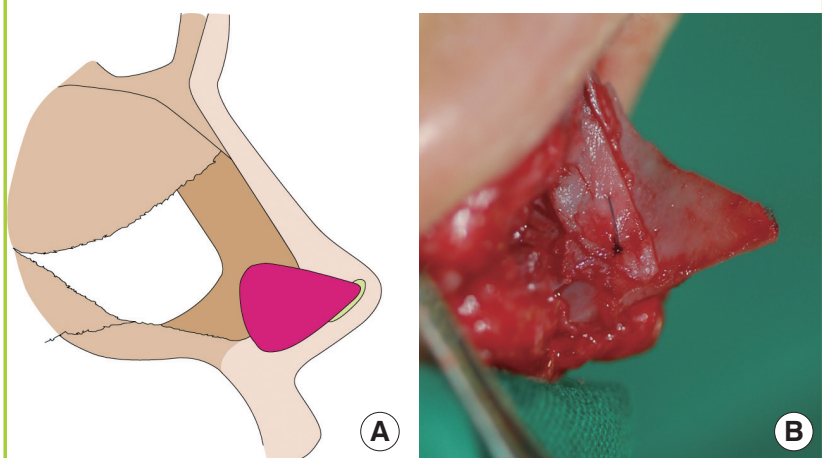

being used was increased compared to other cases. Therefore, the graft was carefully harvested and delicately designed to prevent unnecessary consumption and to avoid the need to harvest from other donor sites. However, the concha cartilage may be used in addition if necessary. Except in cases such as the correction of a severely inclined columella or deviated caudal septum, the process of locating or fixing the graft down to the anterior nasal spine was avoided as much as possible. Last, a type III septal extension graft that provided reinforcement down to the columellar base for strong fixation strength was used to correct the retracted columella. This caused the nasolabial angle to increase with the relatively thicker part of the harvested septal cartilage located frontward, allowing the graft to be located sufficiently downward toward the columellar base (Fig. 5).

For the firm fixation of the graft, the contact surface of the graft and the nasal septum was created to be around 3 to $4 \mathrm{~mm}$ and 


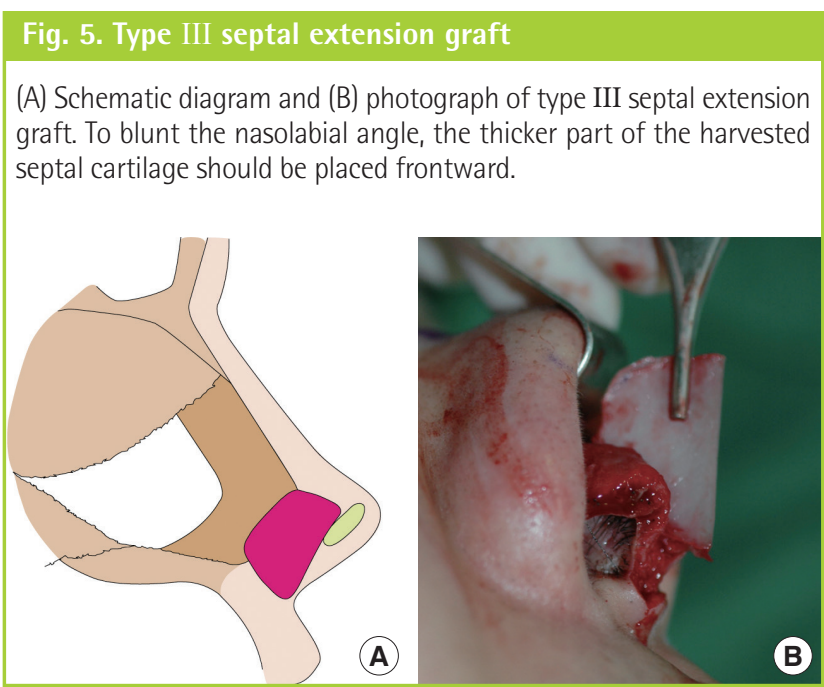

\section{Fig. 6. The unilateral batten graft}

A small unilateral batten graft (arrows) on the other side allows the centralization of the septal extension graft and the efficient use of cartilage.

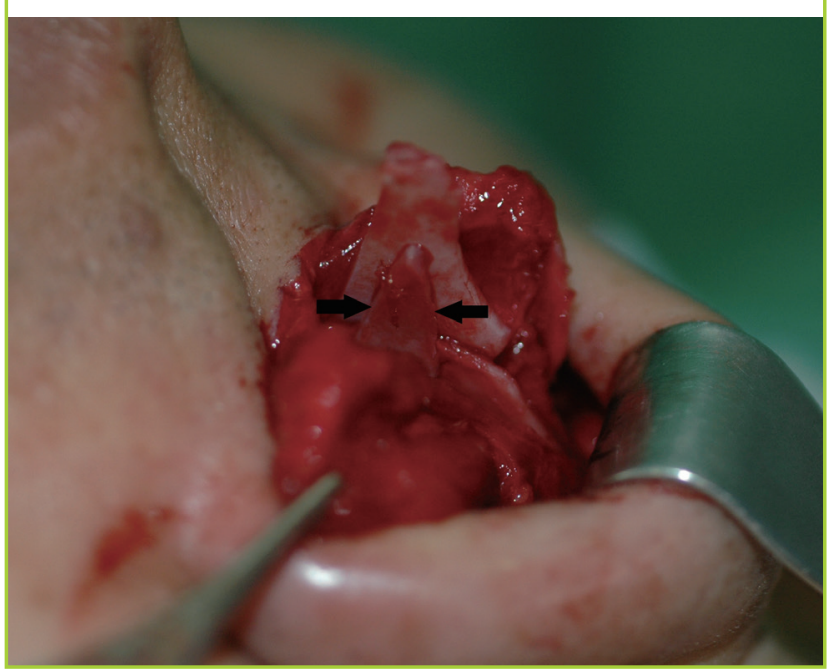

the anchoring suture was performed in more than 4 places. To reduce instability, the pivot locking suture [7] was performed on the dorsal and the caudal parts where the graft and the $\mathrm{L}$ strut meet.

When the septal extension graft is performed on Asians, the efficient use of a relatively small amount of cartilage is needed. Therefore, a relatively large portion of harvested cartilage was allotted to the graft and a small reinforcing batten graft was added on the opposite side to compensate for a potential lack of support (Fig. 6). The batten graft on the opposite side allowed the centralization of the septal extension graft and prevented the gradual deviation of the graft. In addition, other procedures such as a batten graft on the dorsal side (Fig. 7), a columellar strut on the caudal side, and various suture techniques, including the

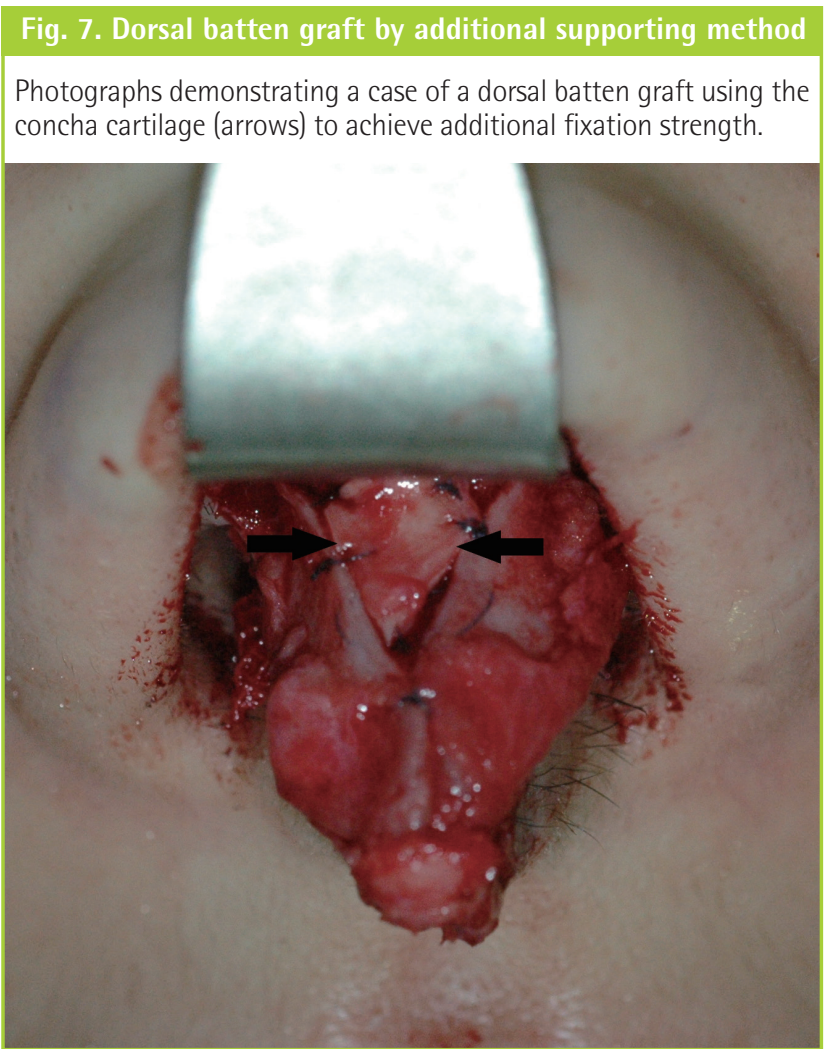

Fig. 8. Unilateral batten graft using the concha cartilage

After a unilateral septal extension graft is placed, an additional batten graft using concha cartilage (arrow) is placed at the contralateral site.

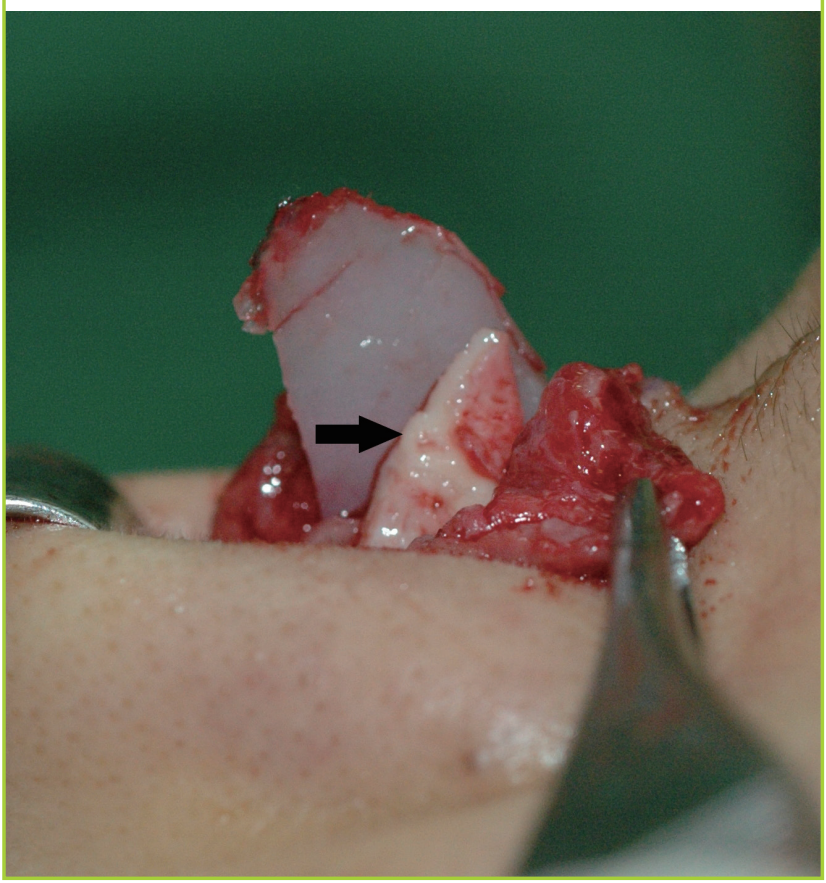

tip extension suture in cases of abundant lower lateral cartilage, were applied when the fixation strength seemed insufficient and additional extension seemed needed. 


\section{RESULTS}

The decision to locate the septal extension graft either on the left or the right side was made, taking into consideration the size of the nostrils and the deviation of the caudal septum. When there was no problem for either side, it was mainly placed on the right side for the surgeon's convenience. A small batten graft was performed on the opposite side of the septal extension graft for all of the patients. Among all of the patients, $11 \mathrm{had}$ a portion of the bony septum harvested along with the sepal cartilage since the amount of cartilage harvested was too small, and 23 patients required an additional harvest of concha cartilage. Of those 23 patients, the harvested concha cartilage was used for the unilateral batten graft for 4 of them (Fig. 8), the dorsal batten graft for 2 of them, and the tip graft for all of them. Among the patients postoperatively followed over 6 months, the average nasal tip angle for the type I septal extension graft patients (upturned tip correction as the main objective) decreased from 124.9 to 98.3 degrees, and the average tip projection increased from 23.5 to $26.9 \mathrm{~mm}$. For the type II septal extension graft patients (tip derotation and projection at the same time), the average tip angle was reduced to 96.8 from 122.4 degrees and the average tip projection was increased to 27.5 from $23.2 \mathrm{~mm}$. The average columella-labial angle for the type III septal extension graft patients (retracted columella correction) was increased to 94.6 from 74.8 degrees (Tables 1,2). The best results for tip mobility were achieved in type I septal extension graft patients and no

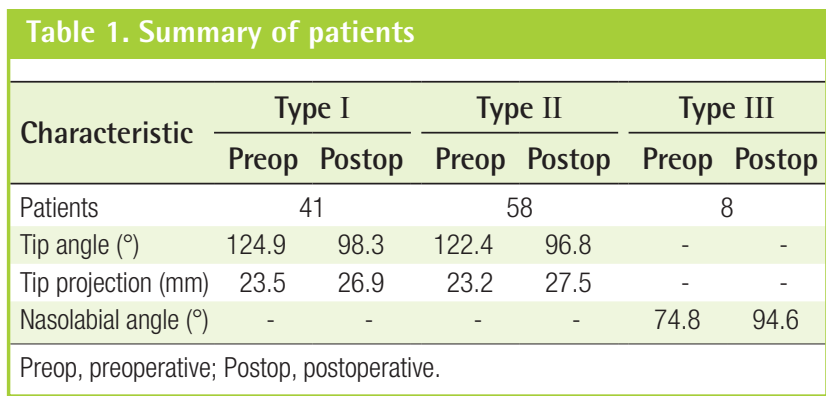

patients of any type of graft reported experiencing discomfort in upper lip motion or clicking sensations while talking or smiling. The patients were followed over a period of 6 to 36 months, with an average of 10 months, and 7 patients ended up having the implant changed due to a foreign body reaction. Most of the patients were satisfied with the nasal tip location or the tip projection and only 5 patients underwent revision operations for additional tip projection at the patients' request. Concerning nasal tip flexibility, no major complaint was reported and the satisfaction level increased with increasing preservation of the membranous septum of the lower portion, which allowed for better flexibility. No other serious complications or noticeable recurrences were observed.

\section{Case 1}

A 28-year-old woman with two prior operations presented with an upturned nose caused by contracture from a prior operation. She underwent the removal of the implant with the capsule and the scar, and a type I septal extension graft was applied to lengthen the nasal tip. After 6 months, the postoperative tip

\begin{tabular}{|c|c|c|}
\hline Classification & Procedures & Patients \\
\hline \multirow[t]{4}{*}{ Type I } & SEG+UBG & 30 \\
\hline & $\mathrm{SEG}+\mathrm{UBGC}$ & 1 \\
\hline & $\mathrm{SEG}+\mathrm{UBG}+\mathrm{TES}$ & 8 \\
\hline & $\mathrm{SEG}+\mathrm{UBG}+\mathrm{CS}$ & 2 \\
\hline \multirow{5}{*}{ Type II } & $S E G+U B G$ & 47 \\
\hline & SEG+UBGC & 2 \\
\hline & $\mathrm{SEG}+\mathrm{UBG}+\mathrm{TES}$ & 7 \\
\hline & $\mathrm{SEG}+\mathrm{UBG}+\mathrm{DBG}$ & 1 \\
\hline & $\mathrm{SEG}+\mathrm{UBGC}+\mathrm{DBG}$ & 1 \\
\hline \multirow[t]{2}{*}{ Type III } & $\mathrm{SEG}+\mathrm{UBG}$ & 6 \\
\hline & SEG+UBG+TES & 2 \\
\hline
\end{tabular}

\section{Fig. 9. Type I septal extension graft (case 1)}

A 28-year-old woman with an upturned nose. A type I septal extension graft was combined with a septal extension suture to rotate her nasal tip. (A, B) Preoperative frontal and right lateral views. (C, D) Postoperative frontal and right lateral views.

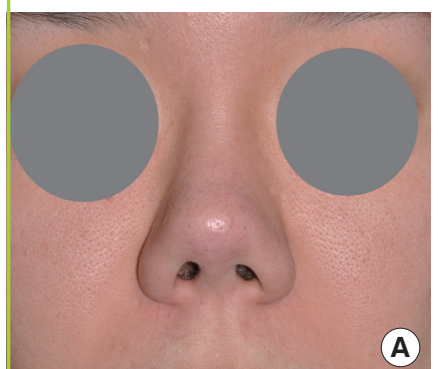

(A)
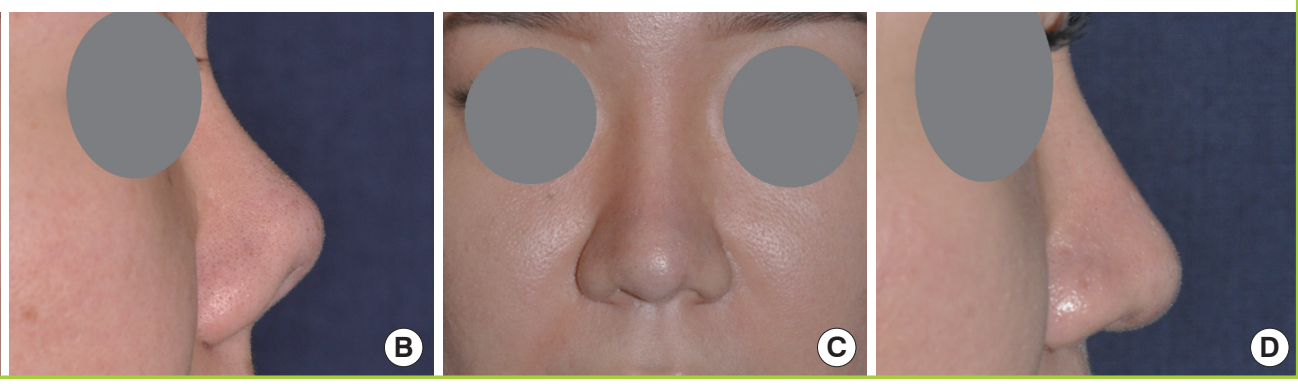


\section{Fig. 10. Type II septal extension graft (case 2)}

A 26-year-old woman who underwent previous augmentation rhinoplasty resulting in an unsatisfactory upturned nose. She underwent the placement of a type II septal extension graft and a contralateral batten graft. She also had a tip onlay graft with dorsal augmentation with a silicone implant. (A,

B) Preoperative frontal and right oblique views. (C, D) 24-month postoperative frontal and right oblique views.
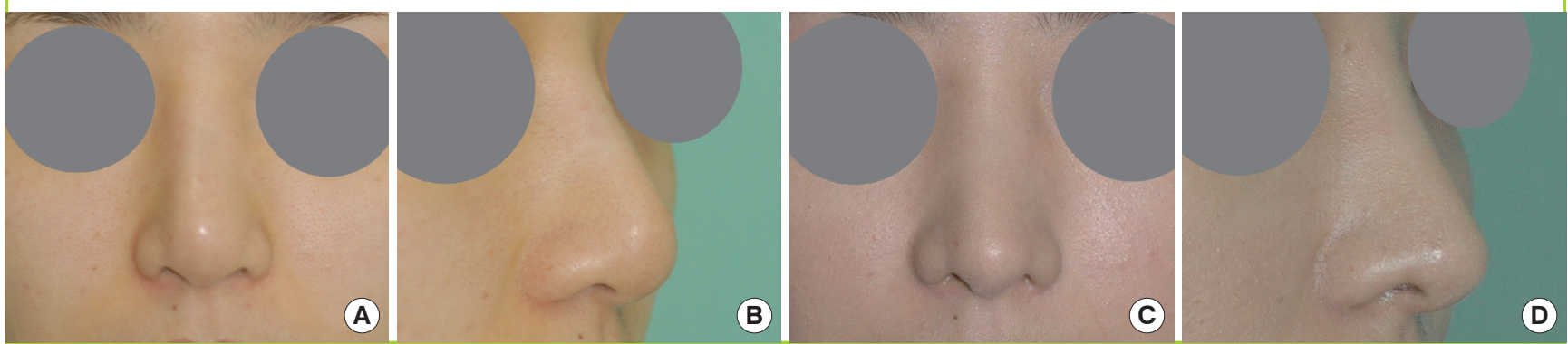

\section{Fig. 11. Type III septal extension graft (case 3)}

A 19-year-old woman with an upturned nose and retracted columella. A type III septal extension graft was used to blunt the nasolabial angle. (A, B) Preoperative right oblique and right lateral views. (C, D) Postoperative right oblique and right lateral views.
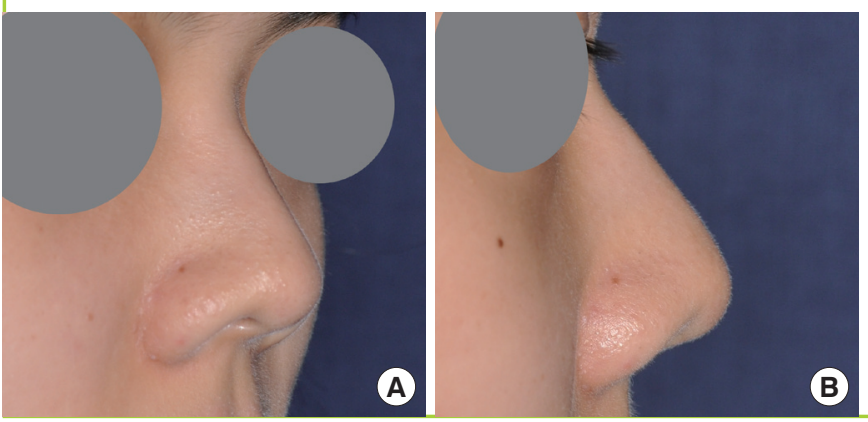

angle had decreased to 98 degrees from a preoperative tip angle of 132 degrees, and the postoperative tip was mobile and natural (Fig. 9).

\section{Case 2}

A 26-year-old woman with two prior operations visited the clinic out of dissatisfaction with the results of prior operations. A type II septal extension graft and tip graft were applied to remove the implant with the capsule and the scar tissue and at the same time conduct an upturned tip correction and projection. A silicone implant was used for dorsal augmentation. The preoperative tip angle was 128 degrees and the nasal height was $27 \mathrm{~mm}$. After 24 months, the postoperative tip angle had decreased to 107 degrees and the nasal height had become $28 \mathrm{~mm}$. In accordance with the patient's preferences, the nasal tip height was not modified (Fig. 10).

\section{Case 3}

A 19-year-old woman with no prior operations presented excessive nostril show from the frontal view and a retracted columella. A type III septal extension graft was applied for the correction of the nasal tip and columellar retraction at the same time. The
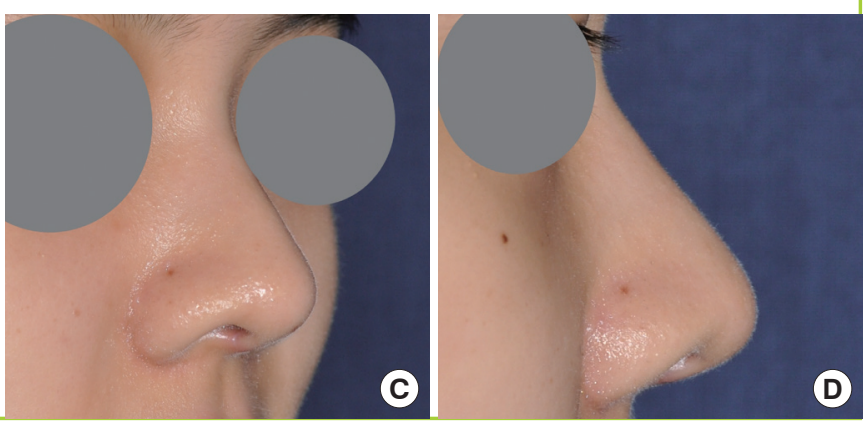

postoperative columella-labial angle after 6 months had increased to 93 degrees from a preoperative columella-labial angle of 79 degrees (Fig. 11).

\section{DISCUSSION}

The economic growth and the accumulation of wealth in Asia brought an expansion of the aesthetic plastic surgery market and increased social acceptance towards it. This change, accompanied by the accumulation of anatomic knowledge and efforts of plastic surgeons to meet the heightened expectations of patients, has led to great development and innovation in both surgical procedures and outcomes. Anatomically, East Asians have different nose structures and characteristics than Caucasians, and nasal tip projection and lengthening for Asians is a major challenge that must be met to achieve satisfactory results. The septal extension graft is a useful procedure for the anatomic characteristics of Asians and has been widely applied in South Korea. However, the disadvantages, such as recurrence, a stiff and rigid nasal tip, a limited amount of septal cartilage, and the overall difficulty of the procedure have caused some surgeons to narrow the eligibility criteria for surgery or perform fewer operations. For this 
reason, we have applied different types of septal extension grafts suited to particular cases and various supplemental methods together to overcome the disadvantages of the septal extension graft and maintain the utmost naturalness of the nasal tip.

Septal extension grafts have been gradually modified and developed. The intercrural strut was efficient for tip projection but had limits in rotation of the nasal tip or correction of the columella-labial angle. To overcome these limits, Tebbetts [8] fixed the columella control strut to the caudal septum for better tip projection, tip rotation, and shape. Byrd et al. [9] introduced 3 types of septal extension grafts (paired spreader grafts, paired batten grafts, and direct extension grafts), depending on the nasal tip position. Although overlapping grafts can provide more support and prevent deviation, they cause a stiff nasal tip and thick membranous septum. Although Seyhan et al. [10] introduced various forms of septal extension graft of a modified batten type to overcome these deficiencies, and Sen and Iscen [11] introduced a caudal septal advancement technique that slides the nasal septum to the front allowing fixation without an overlapping surface, applying these techniques to East Asians is difficult due to the prevalence of a thin caudal septum. Toriumi et al. [12] made a caudal extension graft longer along the superior margin to push the nasal tip down for the counter-rotation of the nasal tip, and made the graft longer along the inferior margin to increase tip rotation and blunt the nasolabial angle. However, these methods require a large amount of cartilage since the graft extends down to the anterior nasal spine and changes the mobile nasal tip to a static structure due to the fixation of the graft to the anterior nasal spine.

Most surgeons using septal extension grafts are concerned about achieving successful outcomes without recurrence while having an insufficient amount of cartilage to utilize. The amount of harvestable septal cartilage is comparatively smaller for East Asians, which makes it especially difficult to apply the various useful methods mentioned above. For cases where using the septum was not possible due to previous rhinoplasty or trauma, Kim and Kim [13] performed short nose corrections with septal extension grafts using rib cartilage. The potential for donor site morbidity and the risk of cartilage warping must be considered when using rib cartilage. Therefore, the efficient use of septal cartilage is essential to avoid additional cartilage harvesting from other sites. We have applied different types of septal extension grafts based on the objective of the operation (upturned tip correction or tip lengthening, simultaneous tip lengthening and nasal tip projection, or retracted columella correction). A graft that conserves the lower portion of the membranous septum as much as possible is used for the cases of upturned tip correction or tip lengthening. This graft allows for the efficient use of cartilage by reducing the amount of septal cartilage being used compared to the conventional septal extension graft, and the preservation of a pliable tip.

The narrow portion at the lower end of the nasal septum, lying between the cartilaginous septum and the columella, is called the membranous septum, or the septum mobile nasi. As its name implies, it is the most flexible part of the septum [14]. The membranous septum consists of the outer layer of vestibule skin, subcutaneous fat, and muscles $[15,16]$. It plays an extremely important role in maintaining nasal tip mobility and is also an important structure involved in facial expression, especially that elicited by speech and laughter. Therefore, tip rigidity can be minimized by preserving the lower portion of the membranous septum as much as possible. For this reason, we partially disagree with the idea that nasal tip rigidity following septal extension grafts is inevitable due to the use of a fixed-type strut and the avoidance of fixing the graft on the entire columella over the entire membranous septum or on the anterior nasal spine. Some may question the maintenance of nasal tip derotation in this graft. We do not think permanent fixation force can be achieved with septal extension grafts and believe that a more important role for the graft is as a strut that is maintained until the formation of a stable biologic scar cast with minimum tension, rather than as a permanent strut $[1,17]$. We have noted that, rather than just focusing on the steady fixation of the graft in upturned nose correction [1], a complete release of the alar cartilage to allow its free movement is absolutely necessary. Also, Jeong [16] mentioned that when the tripod framework is adequately separated for sufficient alar advancement and projection, the amount of cartilage needed can be reduced and even just a simple strut can provide substantial effects. Paik and $\mathrm{Chu}[18]$ mentioned that the methods and the materials for fixing the caudal rotated alar cartilage should possess the minimum amount of strength to cover with the skin flap without distorting the extended cartilage structure. As high skin tension causes excessive wound healing, which leads to keloid or hypertrophic scarring [19], postoperative intranasal tension might cause excessive scarring or scar contracture. Once scar contracture begins, deformity or distortion is inevitable, even with a very strong strut. Therefore, sufficient tension-free release is very important, and as mentioned in the 'surgical technique' above, the complete dissection of the support structure or the scar tissue, holding the nasal tip framework firmly, is the most important step in the tip lengthening procedure. Furthermore, to minimize scar contracture and produce a stable scar cast, a drain, various splints, and compressive dressing were applied for thorough hemostasis and hematoma prevention. Although, in most cases, the fixation strength is reinforced by a small batten 
graft on the opposite side, other procedures were applied as well when the fixation strength seemed insufficient. These included a batten graft on the dorsal side, a columellar strut on the caudal side, and various anchoring sutures, including a tip extension suture in cases with abundant availability of lower lateral cartilage. With at least 6 months of follow-up for the cases of lower membranous septum preservation, although there were some cases of slight projection loss, very few cases of recurrence of derotation or tip lengthening or complaints about unnaturalness due to nasal tip stiffness were reported, and the best possible maintenance of nasal tip mobility was achieved. A graft that sufficiently covers down to the nasal base is used for cases of tip lengthening with tip projection. However, the graft is not pulled down nor fixed to the anterior nasal spine, even in these cases, since it not only makes the nasal tip rigid, but may cause discomfort in upper lip motion or unnaturalness of motion. In addition, it may cause clicking sounds when the nose is being held or a laughing facial expression is made [20]. Although one paper reported observing no such problems in long-term follow-up after complete septal extension grafts [21], the possibility of such occurrences must not be excluded.

Furthermore, since gradual thickening commonly occurs at the connection of the caudal margin of the septum to the premaxilla the farther down it goes, tip deviation may occur when the graft is placed too low near the anterior spine. Although a slight loss of tip projection might results from not locating the graft far enough toward the bottom, the full awareness and realistic expectations of the patients can be attained through preoperative counseling. Furthermore, the actual level of tip projection loss itself was very small according to the results of postoperative follow-up of more than 6 months. These results are assumed to be caused by the fact that the thick skin of East Asians' nasal tip contributes more to the maintenance of the nasal cartilaginous framework than that of Caucasians, and this has played a certain role in the maintenance of nasal tip projection, even after extensive dissections. Another contribution of these results is that the extensive graft piece maintained stability until the formation of the biologic scar cast [17].

However, since loss of projection has occurred compared to the complete septal extension graft, the level of tip projection loss has been estimated and complemented for by overcorrection. Thus, we recommend to patients in preoperative counseling a more mobile nasal tip for naturalness, even it means a slight loss of tip projection.

In the cases of retracted columella correction, the comparatively thicker part of the harvested septal cartilage was placed such that it would come to the front and was designed to be long enough to reach down to the retracted columellar base, allowing the nasolabial angle to be increased while not requiring the graft to extend down to the anterior nasal spine. The septal cartilage becomes thicker, reaching up to 2 to $3 \mathrm{~mm}$, as it extends toward the place where it is attached to the osseous framework of the posterior and lower part, so it was placed on the thicker side, toward the front. In the cases of a shortage of cartilage, an onlay graft with concha cartilage or soft tissue was used to fill the columellar base. In all of the septal extension grafts, instead of being applied to both sides anatomically, it was applied to only one side based on the thickness and the direction of deviation and a small amount of cartilage was added by unilateral batten graft on the other side allowing centralized support and the efficient use of cartilage.

The septal extension graft is a useful method of controlling the projection and rotation of the nasal tip simultaneously, and is a very effective method for changing the weak nasal tip framework of Asians. In order to widen its range of application and maintain the most natural nasal tip, we sought to overcome the septal extension graft's shortcomings through the use of different types of grafts depending on the operation's objective, the complete tension-free release of the alar cartilage, and additional methods. Through these efforts, an optimal nasal tip rotation and projection via the efficient use of minimal septal cartilage was achieved. Also, the biggest disadvantage of the graft, nasal tip stiffness, could be avoided, while maintaining a more pliable nasal tip was achieved by preserving the membranous septum.

\section{REFERENCES}

1. Kim JH, Oh WS, Park SW, et al. Various surgical procedures in the scale of upturned nose. J Korean Soc Aesthetic Plast Surg 2010;16:21-34.

2. Gunter JP, Rohrich RJ, Friedman RM, et al. Importance of the alar-columellar relationship. In: Gunter JP, Rohrich RJ, Adams WP Jr, editors. Dallas rhinoplasty: nasal surgery by the masters. 2nd ed. St. Louis, MO: Quality Medical Pub.; 2007. p. 401-9.

3. Adamson PA, Morrow TA. The nasal hinge. Otolaryngol Head Neck Surg 1994;111:219-31.

4. Rohrich RJ, Hoxworth RE, Thornton JF, et al. The pyriform ligament. Plast Reconstr Surg 2008;121:277-81.

5. Kim JH, Park SW, Oh WS, et al. New classification for correction of alar retraction using the alar spreader graft. Aesthetic Plast Surg 2012;36:832-41.

6. Kim JH. Correction of upturned nose. In: Koeran Society of Rhinoplasty Surgeons, editor. Current trends in Asian rhinoplasty: operation guide. Seoul: Koonja Publishing, Inc.; 2011.p. 349-69. 
7. Oh SH, Kang NH, Woo JS, et al. Stabilization of unilateral septal extension graft using pivot locking suture. J Korean Soc Aesthetic Plast Surg 2008; 14:156-60.

8. Tebbetts JB. Shaping and positioning the nasal tip without structural disruption: a new, systematic approach. Plast Reconstr Surg 1994;94:61-77.

9. Byrd HS, Andochick S, Copit S, et al. Septal extension grafts: a method of controlling tip projection shape. Plast Reconstr Surg 1997;100:999-1010.

10. Seyhan A, Ozden S, Ozaslan U, et al. A simplified use of septal extension graft to control nasal tip location. Aesthetic Plast Surg 2007;31:506-11.

11. Sen C, Iscen D. Caudal septal advancement for nasal tip projection and support in rhinoplasty. Aesthetic Plast Surg 2006;30:135-40.

12. Toriumi DM, Patel AB, DeRosa J. Correcting the short nose in revision rhinoplasty. Facial Plast Surg Clin North Am 2006;14:343-55.

13. Kim SK, Kim HS. Secondary Asian rhinoplasty: lengthening the short nose. Aesthet Surg J 2013;33:353-62.

14. Stovin JS. The importance of the membranous nasal septum.
AMA Arch Otolaryngol 1958;67:540-1.

15. Han SK. Surgical anatomy of Korean nose. J Korean Soc Aesthetic Plast Surg 2005;11:1-9.

16. Jeong JY. Tripod framework rebuilding in Asian nose: tip plasty using alar advancement technique. J Korean Soc Aesthetic Plast Surg 2010;16:125-38.

17. Tebbets JB. Primary rhinoplasty: a new approach to the logic and the techniques. St. Louis, MO: Mosby; 1998.

18. Paik MH, Chu LS. Correction of the short nose using derotation graft. Arch Aesthetic Plast Surg 2012;18:35-44.

19. Mirastschijski U, Jokuszies A, Vogt PM. Skin wound healing: repair biology, wound, and scar treatment. In: Neligan PC, editor. Plastic surgery. 3rd ed. Philadelphia, PA: Elsevier Saunders; 2013. p. 267-96.

20. Bafaqeeh SA. Open rhinoplasty: effectiveness of different tripplasty techniques to increase nasal tip projection. Am J Otolaryngol 2000;21:231-7.

21. Kim JS, Han KH, Choi TH, et al. Correction of the nasal tip and columella in Koreans by a complete septal extension graft using an extensive harvesting technique. J Plast Reconstr Aesthet Surg 2007;60:163-70. 